\title{
Review Study on Digital Image Processing and Segmentation
}

\author{
Puja Shashi ${ }^{1}$, Suchithra $\mathbf{R}^{2}$ \\ ${ }^{1}$ Computer Science Department, Jain University, Bangalore, India \\ ${ }^{2}$ HOD IT Department, Jain University, Bangalore, India
}

Email address:

puja_pramil@yahoo.com (Puja S.),suchithra.suriya@gmail.com (Suchithra R)

\section{To cite this article:}

Puja Shashi, Suchithra R. Review Study on Digital Image Processing and Segmentation. American Journal of Computer Science and Technology. Vol. 2, No. 4, 2019, pp. 68-72. doi: 10.11648/j.ajcst.20190204.14

Received: October 15, 2019; Accepted: November 9, 2019; Published: December 31, 2019

\begin{abstract}
Image segmentation is in fact one of the most fundamental approach of digital image processing. In image processing, segmentation playa an important role. It may be defined as partitioning an image into meaning full regions or objects. In other words we can say that process of segmentation keeps on dividing an image into its constitute sub parts. The level to which the subdivision is carried on depends on type of problem to be solved by researchers. This segmentation process continues unless area of interest is isolated. Set of segment or set of contours that are extracted from the image is the main result of image segmentation. There are various application of image segmentation like locating tumors or other pathologies, measuring tissue volume, surgery aided by computer, treatment and planning, study of various anatomical structure, locating objects in satellite images, fingerprint. There are various types of generalized algorithm and methodology that are developed for image segmentation. Some common technique of image segmentation such as edge detection, thresh holding, region growing and clustering are taken for this study. In fact segmentation algorithm are based on two properties similarity and discontinuity. This paper concentrates on the various methods that are widely used to segment the image.
\end{abstract}

Keywords: Segmentation, Digital Image Processing, Edge Detection, Thres Holding, Region Growing, Clustering

\section{Introduction}

Image segmentation is one of the most important steps in digital image processing. It also involves great area of research in computer vision. The main and foremost objective of image segmentation is to filter and extract region of interest for analysis of image. Hence it may also be defined as division and sub division of an image into multiple structure and sub structure. Segmentation plays a vital role in digital image processing as when the image is subdivided it makes further image analysis easier. Segmentation also depends on various characteristics such as colour or texture. Before removing an error or denoising an image, it is first segmented. Thus segmentation of an image helps in increasing modularity of information.

\section{Types of Segmentation}

Various researchers that fall into given broader classification have proposed a wide range of various types of segmentation.

\subsection{Threshold Based Segmentation}

This is one of the simplest methods of image segmentation. It can be applied either directly to an image or can also be combined with pre and post processing technique. This method can be used to convert black and white or monochrome image can be used to create binary images [1]. In this type of segmentation pixels are given to categories according to range of values in which pixel lies [2]. e.g. suppose an image is to be segmented with pixel less than 128 can be placed in one category and others can be placed in different categories. Further boundary between two adjacent pixels can be superimposed in white or original image. This new image is said to be threshold-segmented image of two predominant types.

Shipping Zhu [3] proposed a new threshold based edge detection and image segmentation algorithm. They in fact calculated threshold of each pixel in the image on the basis of its neighborhood pixels. They also find the edges of image 
with help of proposed algorithm. A threshold of each pixel was set using histogram. PDF is used to separate the background and threshold of an image. They executed their algorithm in visual $\mathrm{C}++$. Results thus obtained outperform the Canny Operators results, because it performs edge detection and segmentation at the same time. Anping XU [4] proposed a threshold based segmentation and Fast marching method (FMM) for medical image segmentation [5]. The result of de-noising filter is passed to FMM for segmentation purpose with the help of threshold based level set technique. They implement their method in $\mathrm{VC}++$ and ITK. After the experiment, results have shown that level set method based on threshold results in clearer, accurate and more perfect segmentation, it also avoid from edge leakage and improve the speed of segmentation

We can broadly classify threshold technique as follows.

\subsubsection{Global Thresholding}

This type of threshold is applicable only when intensity distribution of an object is enough and sufficiently different
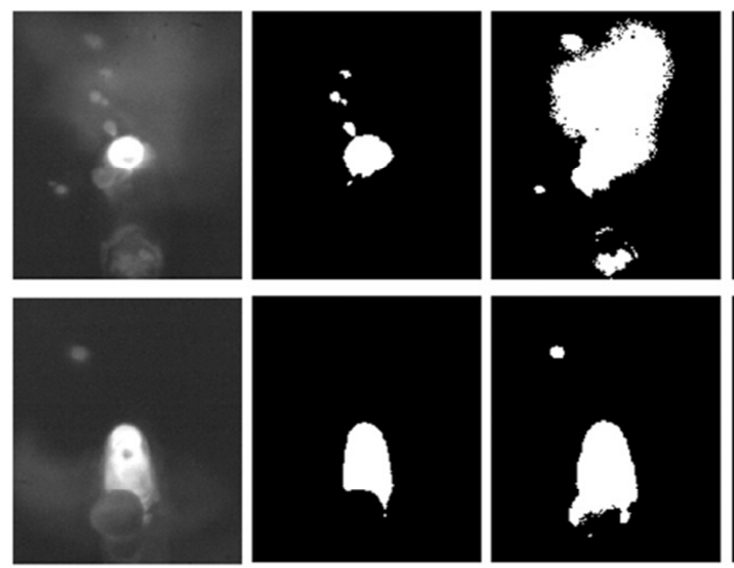

Source

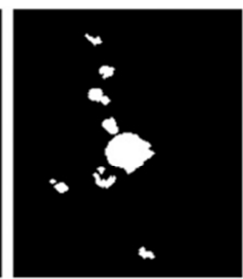

from background pixels. In this case we can define a single threshold values. When $T$ depends only on $f(a, b)$ and this $T$ is only related to the characters of pixels. There are a number of global thresholding techniques such as Otsu, Optimal thresholding, histogram analysis, iterative thresholding, clustering, multispectral and multi thresholding.

\subsubsection{Local Thresholding}

This type of thresholding is applicable when one single value/threshold does not work mainly because of uneven illumination may be due to shadow or due to direction. The main objective is to sub divide the main image into $n \times n$ images and then select the threshold. If threshold depends on both $\mathrm{f}(\mathrm{a}, \mathrm{b})$ and $\mathrm{p}(\mathrm{a}, \mathrm{b})$, then this type of thresholding is called local thresholding. In this type gray level filtering must eliminate gray level discontinuous in sub images. Some example of local thresholding technique are simple statistical thresholding, 2D entropy based thresholding and histogram transformation thresholding.

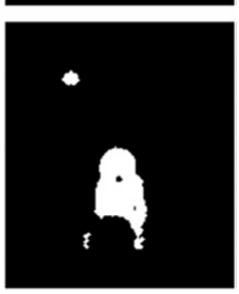

Local threshold

Figure 1. Impact of global and local thresholding.

\subsubsection{Adaptive Thresholding}

This technique takes a colour image as input and outputs binary image. In this technique threshold value of each pixel is calculated, if the value is less than given threshold value, it is taken as background, else it is taken as foreground. This technique is used for separating image object form the background using pixel intensities of various image objects. [6]. The main drawback of this technique is they are computationally expensive and mostly not used in real life application.

\subsection{Edge Based Segmentation}

Edges refer to object boundary. Hence they are problem of fundamental importance in image processing [7]. Edge detection helps in reducing unwanted large amount of data and filtering only important information, thus preserving the important structural property of an image.

Types of proposed edge based segmentation
1. Robert Edge Detection

This is mostly used as an operator in image processing technique. It was first operator that was proposed by Lawrence Robert in 1962. It can perform simple calculation, quickly helps in computation on an image 2D spatial gradient measurement.

\section{Sobel Edge Detection}

This is most commonly used edge detection operator. It is also sometime called as Sobel filter. In the field of image processing Sobel edge detector mostly defines two type of masks.

\section{Horizontal}

3. Vertical

These masks mostly use matrices for edge detection. Sobel edge detector operator defines gradient between horizontal row and vertical column. These operators are mostly used on image that has small, seperable and discrete values.

4. Prewitt Edge Detection

This is one of the oldest and easily understandable edge detector operators. According to it, the magnitude and 
orientation of edge detection need different types of gradient edge detection instead of calculation that is time consuming. This operator has minimum of 8 possible orientations and estimated $3 \times 3$ neighborhood for eight directions.

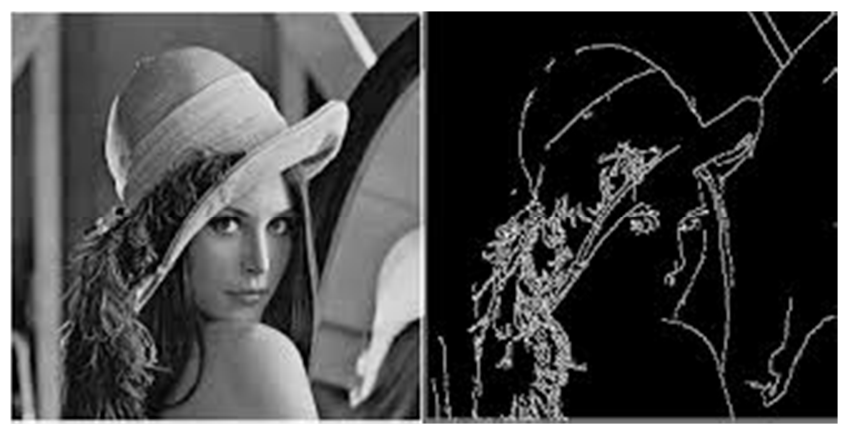

Source: Research gate.

Figure 2. Edge based segmentation.

\subsection{Region Based Segmentation}

It takes the reverse approach as taken by Edge based segmentation. Instead starting at the edge as in previous segmentation, it starts from the middle and slowly grows towards boundary. In this technique pixels that are related to object are grouped for further segmentation. It's very important that area that is detected for segmentation should be closed. This technique is also called similarity-based segmentation. Boundaries in particular are identified for segmentation. In each and every step at least one pixel related to the region should be taken into consideration. Further after identifying the change in colour and texture, the edge flow is converted into a vector [8]. Edge obtained is used for further segmentation.

In this region-based $[9,10]$ segmentation, we segment the similar image into various regions. It is also used to determine the region directly. Using grey values of the image pixels does partitioning. Two basic-type techniques of region-based segmentation are the following:

Region Growing method: It is region-based segmentation scheme in which pixels group or sub regions are changed into large size regions predefined criteria. In this region, growing pixels begin with set of seed points, and in this approach the corresponding regions grow via appending every seed points; those neighboring pixels have related similar properties like greyscale, colour, texture, shape, etc.

Splitting and Merging: In this method, image is taken as a single region and then repeatedly breaks down the image until no further breakdown is possible. After the splitting process, merging process takes place. In merging technique, region is merged with the adjacent regions. The method starts with small regions and then merges the regions, which have similar characteristics like greyscale and variable. Quadtree (Splitting data structure) is used here for merging regions if they are similar and adjacent the merging technique is repeated until max possible merge.
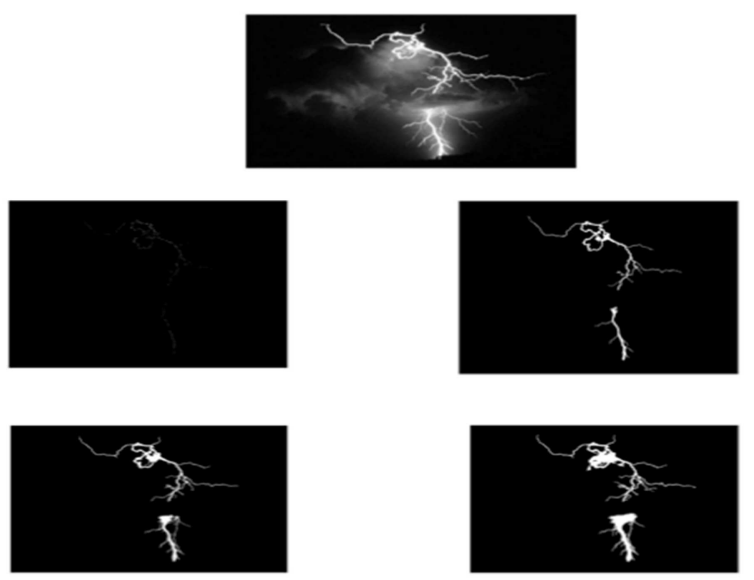

Source: Science direct.com.

Figure 3. Region based Segmentation on the basis various thresholding technique.

\subsection{Morphological Method}

In image processing domain morphology is a term given to identify and analyze geometric inherent structure inside an object. This method checks and verify the image with a small pre defined template called structuring element, that can be applied to all possible location of input image and gives the output of same size. A new binary image is produced by this operation in which if test is successful, it will have non-zero pixel value at that location in an input image. There are various categories of structuring element such as diamond shaped, cross-shaped, and square shaped. Dilation, erosion, opening and closing are the base of morphological operation. Morphological operation can be the base for constructing morphological filtering that are more suitable for analyzing the shape than standard linear filter. Another type of morphology-based technique is morphology watershed that is not made up of primitive morphological operation. H Disable and $\mathrm{C}$ lantuejuel gave the initial concept of watershed tool. Watershed mostly represents the boundary between adjacent catchment. If gray level at each point of an altitude is combined we can define watershed as the bridge between two watershed's boundaries.
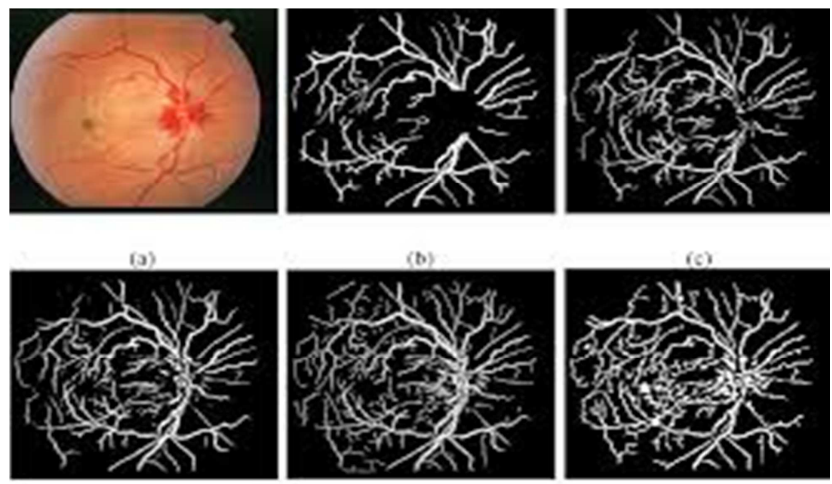

(d)

(c)

in

Source: Journals.plos.org.

Figure 4. Morphological based segmentation. 


\subsection{Model Based Segmentation}

This segmentation is also known as Markov Random Field (MRF). In this technique inbuilt region condition are used for colour segmentation. Various component of colour pixel can be considered as independent variables that can be used for further processing. This technique when combined with Edge detection can detect and edge clearly and precisely [11].

\subsection{Clustering Technique}

Clustering technique can also be used for image segmentation. Some basic algorithm such as K- means is used for mostly for textured image. It groups/clusters the related pixel for segmenting the image. In this case segmentation entirely depends upon characteristic of image. Fuzzy clustering technique [10] is another method that is mostly used for colour image. In this method clustering technique are used that gives colour clusters using fuzzy membership function in colour space with respect to image space. This technique is very helpful in recognizing the coloured region. After getting the image coarsely segmented using multithresholding and is then further refine by Fuzzy c - means clustering, it can be applied to any types of multispectral images. Clustering method some time try to group together pattern that are somewhat similar, hence it is considered as one of the most powerful tool of image segmentation. Analysis is further done to subdivide image data sets into a number of clusters. $\mathrm{K}$ means clustering is one of the most popular methods because of its simplicity and efficiency in computation [12].

Clustering [13] is most popular algorithm in image segmentation. It classifies data and patterns in categories. Two popular examples of clustering methods are K-means and fuzzy c-means [13]. Both methods are capable to produce divide of images under conditions including cluster number and for recovering cluster algorithm, we find out cluster number and centers depend on decision graphs.

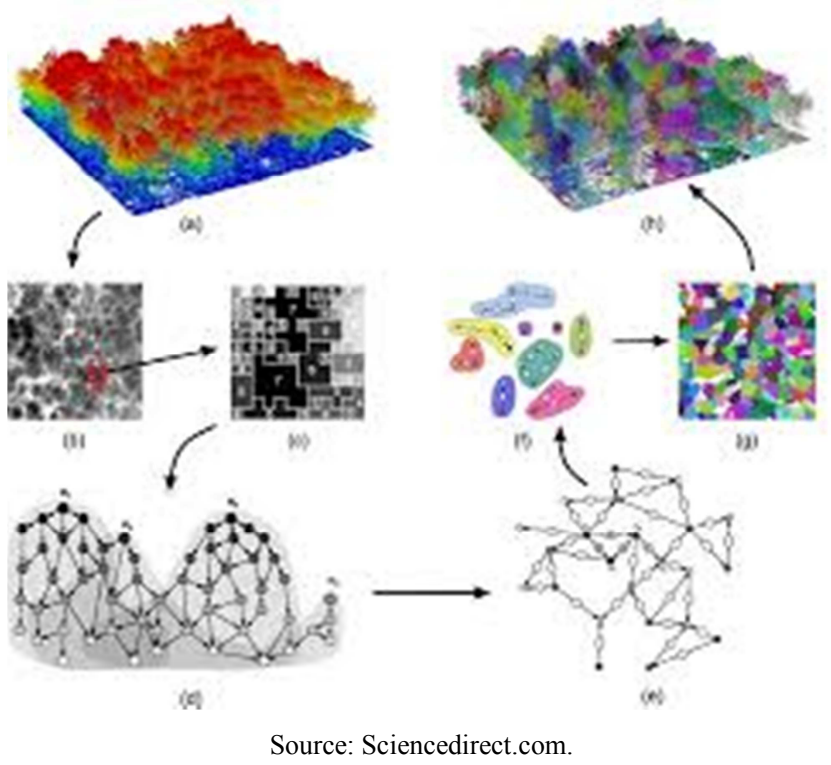

Source: Sciencedirect.com

Figure 5. Clustering based segmentation.

\section{Literature Survey}

This section introduces the related work of the image segmentation by various authors with different perceptions regarding to the segmentation techniques.

Satish Kumar et. al [14], in his survey explained the various applications that uses the concept of the image segmentation which includes, computer vision, medical, scanning, recognition, etc.

P. Sravani et al. [15], in their survey, an overview of different segmentation methods and clustering are studied. Though many techniques are developed, not all types are useful for all types of images. Segmentation segments the image and clusters according to some similarity. Distance metric is a similarity measure and has direct impact on the clusters formed. In this, Fuzzy is powerful unsupervised clustering method which is widely used for robust segmentation of real time images. Traditional FCM and many other algorithms use Euclidean Distance metric.

H. P. Narkhede [16], in his review of image segmentation study, has described various methodologies and issues regarding to digital image processing used in various recognition patterns.

Sujata Saini and Komal Arora Rajeshwar Dass et al. [17], In this paper they classifies and discussed main image segmentation algorithms and concluded that the methods are classified on the basis of the features as: homogeneity of images, spatial characteristics of the image continuity, texture, image content.

Nikita Sharma et al. [18], in their study, reviewed out that the performance assessment and comparison is not easy for the various segmentation techniques or methodologies. They have mentioned the evaluation techniques so that researchers could use while opting any segmentation method.

PunamThakare [19], in her paper describes the various image segmentation techniques and discusses in detail the edge detection techniques and their evaluation. It gives an algorithm which is a combination of detection and evaluation of the edge detectors. The results show that the recognition rate depends on the type of the image and their ground truths.

Dey et. al. [20], in the paper summarized the segmentation methods with the help of modeling approach. Image segmentation methodologies were categorized in three stages. At first stage comes model driven approach and image driven approach (mainly based on statistical analysis). The second stage corresponds to homogeneity based measure, and final category corresponds to mode of operations on an image, e. g. edge detection, region growing/splitting. The selection of segmentation approach depends on what quality of segmentation is required. Further, it also depends on what scale of information is required.

N. Senthilkumaran and R. Rajesh [21], in their paper, mainly focused on the study of soft computing approach to edge detection for image segmentation. The soft computing approaches namely, fuzzy based approach, Genetic algorithm based approach and Neural network based approach is applied on a real life example image of nature scene and the 
results show the efficiency of image segmentation.

\section{Conclusion}

In this paper, we tried to discuss and evaluate main image segmentation technique used for the work of analyzing the image. However it is analyzed that there is as such no perfect method for image segmentation as the result of image segmentation may depend son many factors such as pixel colour, texture, intensity, image similarity, content and also on problem domain. Hence sometimes it is not possible to consider a single method for each and every images also neither all the methods can perform well for a particular type of image. Hence it is always recommended to use hybrid solution that may consist of more than one method for image segmentation problem. Whenever we need to analyze the internal part of, it can be performed by image segmentation. Therefore it forms an important step of image processing. We can apply segmentation to any type of image. When we compare with other method, thresholding can be considered as simplest and fastest method. Segmentation of an image can be performed according to application requirement. As already mentioned segmentation method are classified on the basis of discontinuity and similarity of the image. According to study, Markov field impose strong spatial constraints on the segmented regions, whereas when segmentation depends on only pixel intensities. Hence HMRF segmentation results are much more direct and easier than the result of K-means clustering.

\section{References}

[1] Shapiro, Linda G. \& Stockman. George C. (2002). "Computer vision". Prentice Hall. ISBN 0-13-030796-3.

[2] P. Singh, P and R. S. Chadha, A Novel Approach to Image Segmentation, International Journal of Advanced Research in Computer Science and Software Engineering Research Paper, 2013.

[3] S. Zhu, X. Xia, Q. Zhang, and K. Belloulata, "An image segmentation algorithm in image processing based on threshold segmentation," in Proc. Third International IEEE Conference on Signal-Image Technologies and Internet-Based System, SITIS'0., pp. 673-678, 2007.

[4] A. Xu, L. Wang, S. Feng, and Y. Qu, "Threshold-based level set method of image segmentation," in Proc. 3rd International Conference 92 International Journal of Future Computer and Communication, Vol. 3, No. 2, April 2014 on Intelligent Networks and Intelligent Systems (ICINIS), pp. 703-706, 2010.

[5] M. Yasmin, M. Sharif, S. Masood, M. Raza, and S. Mohsin, "Brain image enhancement-A survey," World Applied Sciences Journal, vol. 17, pp. 1192-1204, 2012.

[6] E. R. Davies, Machine Vision: theory, algorithms, practicalities, 1997.

[7] R. Gurcan, I. Erer and S. Kent, An edge detection method using 2-D autoregressive lattice prediction filters for remotely sensed images, In Geoscience and Remote Sensing Symposium, 2004, IGARSS'04, Proceedings of 2004 IEEE International, IEEE, September, 2004, Vol. 6, pp. 4219- 4222.
[8] R. Yogamangalam and B. Karthikeyan, Segmentation techniques comparison in image processing, International Journal of Engineering and Technology (IJET), 2013, 5 (1), 307-313.

[9] J. Serra and P. Salembier, "Connected operators and pyramids," in Image Algebra and Morphological Image Processing IV, vol. 2030 of Proceedings of SPIE, San Diego, Calif, USA, July 1993, pp. 65-76.

[10] Senthilkumaran, N., Rajesh, R.: Edge detection techniques for image segmentation-A survey of soft computing approaches. Int. J. Recent Trends Eng. 1 (2), 250-254 (2009).

[11] Hore, S., et al.: An integrated interactive technique for image segmentation using stack based seeded region growing and thresholding. Int. J. Electr. Comput. Eng. 6.6, 2773 (2016) 9. Smistad, E., et al.: Medical image segmentation on GPUs-A comprehensive review. Med. Image Anal. 20.1, 1-18 (2015).

[12] D. Ziou and S. Tabbone, Edge detection techniques-an overview, Pattern Recognition and Image Analysis $\mathrm{C} / \mathrm{C}$ of Raspoznavaniye Obrazov I Analiz Izobrazhenii, 8, 1998, 537-559.

[13] T. Kanungo, D. M. Mount, N. S. Netanyahu, C. D. Piatko, R. Silverman, and $\mathrm{A}$. Y. Wu, An efficient k-means clustering algorithm: Analysis and implementation. IEEE transactions on pattern analysis and machine intelligence, 2002, 24 (7), 881-892.

[14] Chen, Z. et al.: Image segmentation via improving clustering algorithms with density and distance. Proc. Comput. Sci. 55, 1015-1022 (2015).

[15] Satish Kumar, Raghavendra Srinivas, "A Study on Image Segmentation and its Methods", International Journal of Advanced Research in Computer Science and Software Engineering, Volume 3, Issue 9, September 2013.

[16] P. Sravani et al, “ A Survey on Image Segmentation Techniques and Clustering”, International Journal of Advance Research in Computer Science and Management Studies, Special Issue, December 2013.

[17] H. P. Narkhede, "Review of Image Segmentation Techniques", International Journal of Science and Modern Engineering (IJISME) ISSN: 2319-6386, Volume-1, Issue-8, July 2013.

[18] Rajeshwar Dass, Priyanka, Swapna Devi, "Image Segmentation Techniques", IJECT Vol. 3, Issue 1, Jan.-March 2012. 1452 Sujata Saini and Komal Arora.

[19] Nikita Sharma, Mahendra Mishra, Manish Shrivastava, "COLOUR IMAGE SEGMENTATION TECHNIQUES AND ISSUES: AN APPROACH", International Journal of Scientific \& Technology Research Volume 1, Issue 4, May 2012.

[20] PunamThakare, "A Study of Image Segmentation and Edge Detection Techniques", International Journal on Computer Science and Engineering (IJCSE), Vol. 3 No. 2 Feb 2011.

[21] V. Dey, Y. Zhang, M. Zhong, "A REVIEW ON IMAGE SEGMENTATION TECHNIQUES WITH REMOTE SENSING PERSPECTIVE", July 5-7, 2010, IAPRS, Vol. XXXVIII, Part 7A.

[22] N. Senthilkumaran and R. Rajesh, "Edge Detection Techniques for Image Segmentation - A Survey of Soft Computing Approaches", International Journal of Recent Trends in Engineering, Vol. 1, No. 2, May 2009. 